\title{
Traumatismo recorrente na dentição decídua e suas implicações: relato de caso
}

Traumatismo recurrente en la dentición caduca y sus implicaciones: reporte de caso Recurrent trauma to the deciduous dentition and its implications: a case report

Laura Imbriani BENTO ${ }^{1}$

Carla Oliveira FAVRETTO

Marcelle DANELON ${ }^{3}$

${ }^{1}$ Departamento de Odontologia, Universidade de Ribeirão Preto, UNAERP, 14096-900 Ribeirão Preto - SP, Brasil ${ }^{2}$ Departamento de Odontopediatria, Faculdade Morgana Potrich, FAMP, Mineiros - GO, Brasil

${ }^{3}$ Departamento de Odontologia, Universidade de Ribeirão Preto, UNAERP, 14096-900 Ribeirão Preto - SP, Brasil; Departamento de Odontologia Preventiva e Restauradora, Faculdade de Odontologia, UNESP Univ. Estadual Paulista, 16015-050 Araçatuba - SP, Brasil

\section{Resumo}

O objetivo do presente estudo foi relatar um caso clínico de subluxação, seguida de trauma recorrente, bem como complicações clínicas, radiográficas e plano de tratamento. Paciente do gênero feminino, 4 anos e 5 meses de idade, compareceu à clínica de Odontopediatria da Faculdade de Odontologia de Araçatuba (FOA/UNESP), Brasil, cuja mãe relatava o aparecimento de "bolinha" na região superior do dente 51 e 61. Durante anamnese, a mãe relatou que a criança havia caído há 12 meses e batido o dente 51 apresentando sangramento, leve mobilidade e fratura corono-esmalte, e que segundo a odontopediatra que atendeu a criança no momento do trauma, se tratava de subluxação, e há 2 meses houve trauma recorrente. Ao exame clínico observou-se alteração na coloração do dente 51 associado à fratura corono-esmalte, fístula e mobilidade. Já no dente 61 , presença de fístula e mobilidade. A análise radiográfica mostrou reabsorção radicular extensa do dente 51 e 61, ambos com rompimento da cripta óssea do germe dos dentes permanentes 11 e 21 , associado a lesão periapical, denotando necrose pulpar. O plano de tratamento instituído foi a exodontia dos dentes 51 e 61 , seguido pelo acompanhamento clínico e radiográfico. Embora a subluxação não seja caracterizada como um trauma severo, é importante informar aos pais/responsáveis pela criança sobre ao acompanhamento longitudinal, uma vez que no futuro consequências mais severas podem ocorrer, e dessa forma quando diagnosticada precocemente, a alteração, é passível de tratamento efetivo e satisfatório, levando à preservação do dente até o momento de sua esfoliação.

Descritores: Traumatismos Dentários; Fístula; Criança.

\section{Abstract}

The aim of the present study was to report a clinical case of subluxation, followed by recurrent trauma, as well as clinical, radiographic complications and treatment plan. Female patient, 4 years and 5 months old, attended the clinic of Odontopediatria of the Faculty of Dentistry of Araçatuba (FOA/UNESP), Brazil, whose mother reported the appearance of a "ball" in the upper region of teeth 51 and 61 . During anamnesis, the mother reported that the child had fallen 12 months ago and hit tooth 51 with bleeding, slight mobility and crown-enamel fracture, and that according to the pediatric dentist who attended the child at the time of the trauma, it was subluxation, and 2 months ago there was recurrent trauma. Clinical examination revealed changes in the color of tooth 51 associated with a corona-enamel fracture, fistula and mobility. In tooth 61 , there was a fistula and mobility. Radiographic analysis showed extensive root resorption of teeth 51 and 61, both with disruption of the bone crypt of the germ of permanent teeth 11 and 21 , associated with periapical injury, denoting pulp necrosis. The treatment plan established was the extraction of teeth 51 and 61 , followed by clinical and radiographic monitoring. Although subluxation is not characterized as a severe trauma, it is important to inform the parents/guardians of the child about longitudinal follow-up, since in the future, more severe consequences may occur, and thus, when diagnosed early, the change is amenable to treatment effective and satisfactory, leading to tooth preservation until the moment of its exfoliation.

Descriptors: Tooth Injuries; Fistula; Child.

\section{Resumen}

El objetivo del presente estudio fue informar un caso clínico de subluxación, seguido de trauma recurrente, así como complicaciones clínicas, radiográficas y plan de tratamiento. Una paciente de 4 años y 5 meses asistió a la clínica de Odontopediatria de la Facultad de Odontología de Araçatuba (FOA / UNESP), Brasil, cuya madre informó la aparición de una "bola" en la región superior de los dientes 51 y 61 . Durante la anamnesis, la madre informó que el niño se había caído hace 12 meses y golpeó el diente 51 con sangrado, movilidad leve y fractura de esmalte corono, y que según el dentista pediátrico que atendió al niño en el momento del trauma, fue subluxación, y Hace 2 meses hubo traumas recurrentes. El examen clínico reveló cambios en el color del diente 51 asociado con una fractura de corona-esmalte, fístula y movilidad. En el diente 61 , había una fístula y movilidad. El análisis radiográfico mostró una extensa reabsorción radicular de los dientes 51 y 61 , ambos con alteración de la cripta ósea del germen de los dientes permanentes 11 y 21 , asociada con lesión periapical, que denota necrosis pulpar. El plan de tratamiento establecido fue la extracción de los dientes 51 y 61 , seguido de monitoreo clínico y radiográfico. Aunque la subluxación no se caracteriza como un trauma severo, es importante informar a los padres / tutores del niño sobre el seguimiento longitudinal, ya que en el futuro pueden ocurrir consecuencias más graves y, por lo tanto, cuando se diagnostica temprano, el cambio es susceptible de tratamiento. eficaz y satisfactorio, que conduce a la preservación de los dientes hasta el momento de su exfoliación.

Descriptores: Traumatismos de los Dientes; Fístula; Niño.

INTRODUÇÃO

A ocorrência de traumatismos é muito frequente na dentição decidua na fase préescolar e escolar, visto que a pouca idade da criança, juntamente com sua falta de coordenação motora e ausência de percepção aos riscos, the oferece muitos prejuízos para, principalmente, os dentes anteriores superiores. A falta de selamento labial da região e a sobressaliência desses elementos dentais, devido à hábitos de sucção recorrentes, são as principais causas de serem os elementos dentários mais afetados frente à eventualidade do trauma ${ }^{1,2}$.

Existe maior predominância de traumatismos dentários em pacientes do sexo masculino, sendo a faixa etária de 2 a 4 anos a mais afetada por fatores que levam ao trauma dental, como, por exemplo: a prática de 
esportes, acidentes automobilísticos, violência, queda, entre outros, que podem atingir não somente dentes anteriores, mas dentes posteriores em igual proporção ${ }^{3}$.

O traumatismo dental pode ser classificado como: concussão, subluxação, luxação lateral, intrusiva ou extrusiva e avulsão ${ }^{4}$. Quando ocorre uma fratura, a lesão pode ocorrer das seguintes formas: fratura de esmalte (com perda de estrutura dentária restrita ao esmalte); fratura de esmalte e dentina (sem exposição pulpar); fratura de esmalte e dentina com exposição pulpar; fratura coronorradicular (envolvendo esmalte, dentina e cemento); fratura radicular (envolvendo esmalte, dentina, cemento e polpa) e fratura alveolar (envolvendo ossos alveolares e adjacentes) ${ }^{5}$.

Nos casos de lesões traumáticas que envolvem os tecidos de sustentação, temos: concussão (de pequena intensidade, sem deslocamento e mobilidade do dente); subluxação (de intensidade moderada, sem deslocamento, mas com mobilidade do dente, sangramento gengival e sensibilidade ao toque); luxação lateral (de maior intensidade, com deslocamento do dente para palatina, vestibular, mesial ou distal); luxação intrusiva (deslocamento axial do dente para o interior do alvéolo, com leve infra-oclusão ou totalmente incluso e edema nos tecidos moles); luxação extrusiva (deslocamento parcial do dente para fora do alvéolo) e avulsão (deslocamento total do dente para fora do alvéolo ${ }^{6,7}$.

A subluxação é considerada como um traumatismo de baixa a moderada intensidade, que afeta os tecidos de sustentação, provocando mobilidade, mas sem causar deslocamento do elemento dental. É muito comum também nestes casos de trauma as crianças apresentarem sangramento gengival e sensibilidade ao toque e à mastigação. Quanto aos exames complementares, não há notificação de achados radiográficos ${ }^{8}$. Quando os tecidos de sustentação dos dentes decíduos são afetados, visto que há alta prevalência de deslocamento devido à maior resiliência do osso alveolar, a escolha de tratamento é diferente para cada tipo de traumatismo. Para concussão, o profissional deve orientar aos pais que haja um controle dietético, com alimentos mais pastosos e líquidos durante os dois primeiros dias após o trauma, evitando o uso de mamadeiras e/ou chupetas. Para subluxação, a orientação dietética e de hábitos de sucção são as mesmas, podendo, em casos de mobilidade dental acentuada, lançar mão de contenção semirrígida por 14 dias, sendo realizado o controle de 4 em 4 meses $^{9}$. É importante que, após o trauma, o dente lesionado seja removido de oclusão, fornecendo repouso para a região traumatizada, além de avaliar a necessidade de medicação (analgésico, antiinflamatório e/ou antibiótico) ou de vacina antitetânica ${ }^{10}$.

O manejo frente ao traumatismo em dentes decíduos é decisivo para o sucesso do tratamento como um todo, devido ao envolvimento do ápice do elemento decíduo com o germe dental permanente successor. Dessa forma, mostra-se extremamente importante o tratamento imediato proposto pelo profissional para evitar possíveis repercussões adicionais ao próprio dente afetado e/ou ao permanente. Dentre as medidas que devem ser realizadas, ressalta-se a importância da anamnese, para que o profissional compreenda "como", "quando" e "onde" aconteceu o trauma, acurado exame extra e intra-bucal, bem como providenciar as radiografias necessárias ${ }^{11}$.

Ainda, sabe-se que o incidente que leva ao trauma é extremamente estressante e assustador, tanto para a família quanto para o próprio paciente. Por isso é de suma importância a calma do odontopediatra e a correta escolha do tratamento de acordo com a maturidade e nível de cooperação da criança ${ }^{12}$.

O objetivo do presente estudo foi relatar um caso clínico de subluxação, seguida de trauma recorrente, bem como complicações clínicas, radiográficas e plano de tratamento.

\section{CASO CLÍNICO}

Paciente do gênero feminino, 4 anos e 5 meses de idade, compareceu à Clínica de Odontopediatria da Faculdade de Odontologia de Araçatuba (FOA/UNESP), Brasil, cuja mãe relatava o aparecimento de "bolinha" na região superior do dente 51 e 61 , sendo acompanhado de fratura corono-esmalte (Figura 1).

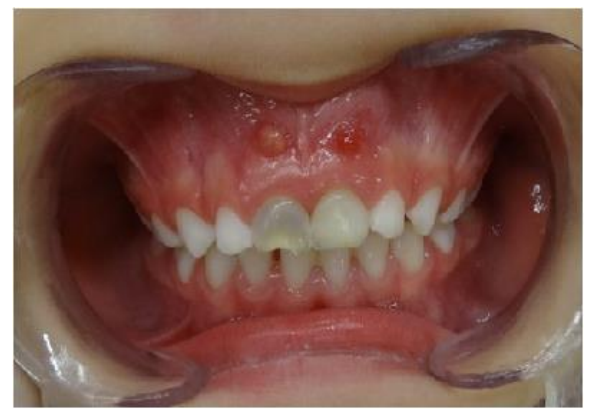

Figura 1: Aspecto clínico inicial, vista frontal.

Durante anamnese, a mãe relatou que a criança havia caído há 12 meses e batido o dente 51 apresentando sangramento, leve mobilidade e fratura corono-esmalte, e que segundo a odontopediatra que atendeu a criança no momento do trauma, se tratava de um traumatismo do tipo subluxação, sendo a conduta o acompanhamento clínico e 
radiográfico e há 2 meses havia ocorrido novo trauma.

Ao exame clínico observou-se alteração na coloração do dente 51 associado à fratura corono-esmalte, fístula e mobilidade. Já no dente 61 foi verificado presença de fístula e mobilidade, sem alteração de cor (Figura 1).

A análise radiográfica mostrou reabsorção radicular extensa do dente 51 e 61, ambos com rompimento da cripta óssea do germe dos dentes permanentes 11 e 21, associado a lesão periapical, denotando necrose pulpar (Figura 2). O plano de tratamento instituído foi a exodontia dos dentes 51 e 61, seguido pelo acompanhamento clínico e radiográfico. Optou-se, inicialmente, pela anestesia tópica da área com EMLA ${ }^{\circledR}(2,5 \%$ Lidocaína e 2,5\% Prilocaína) por 5 minutos. Em seguida, realizou-se a anestesia terminal infiltrativa com Citocaína à 3\% (Figura 3), sindesmotomia com Hollemback 3s (Figura 4), adaptação do Fórceps no 1 aos dentes, seguido das exodontias (Figuras 5 e 6 ).

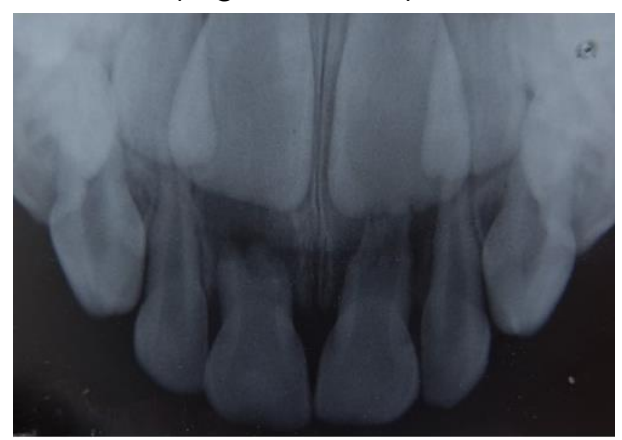

Figura 2: Aspecto radiográfico inicial.

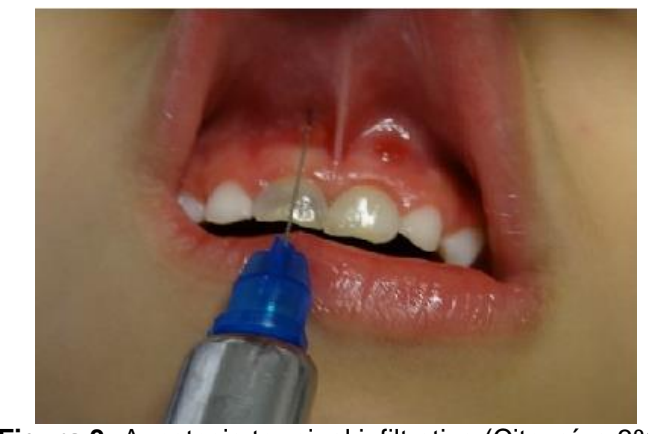

Figura 3: Anestesia terminal infiltrativa (Citocaína 3\%).

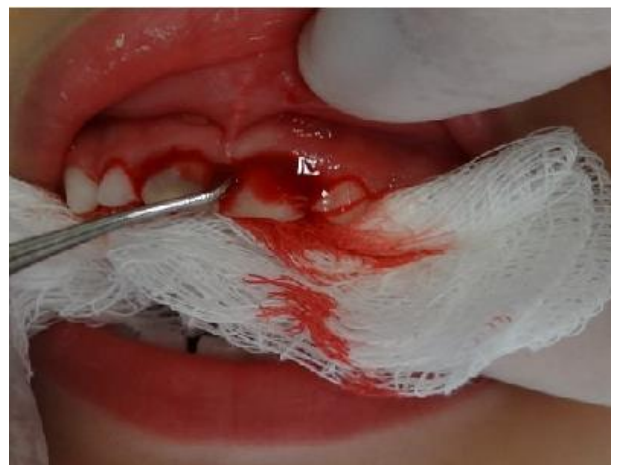

Figura 4: Sindesmotomia com hollemback 3s na região dos dentes 51 e 61 .

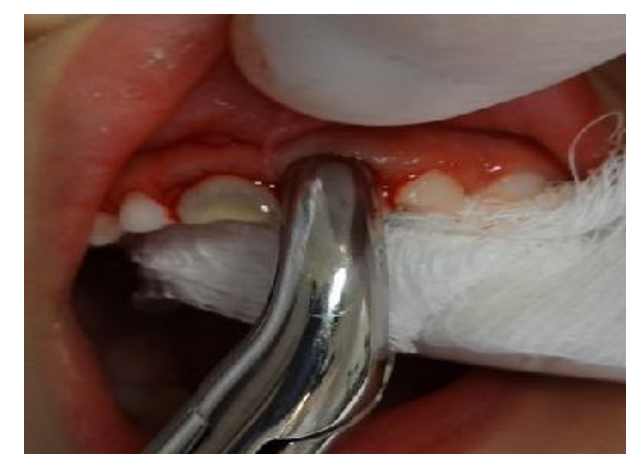

Figura 5: Exodontia com fórceps infantil nº 01 dos dentes 51 e 61 .

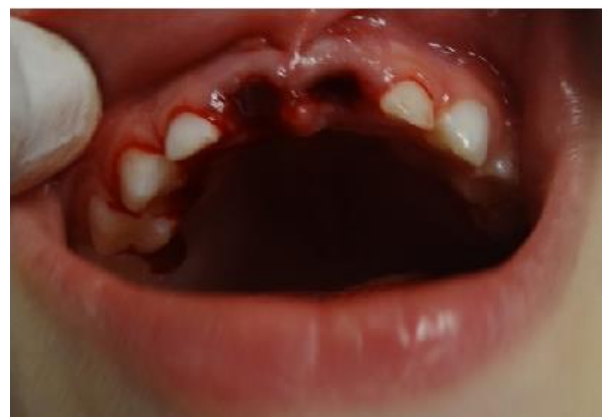

Figura 6: Aspecto clínico após as exodontias (vista frontal).

DISCUSSÃO

Lesões traumáticas na dentição decídua desencadeiam sérios problemas que podem afetar a vida da criança em diversos aspectos, sendo a mesma submetida à uma experiência traumática em nível físico, emocional e psicológico. Quanto mais jovem for o paciente, o medo e a ausência de cooperação serão desafios a serem enfrentados para realizar o exame clínico/radiográfico e 0 devido tratamento ${ }^{9}$.

As crianças são comumente afetadas por lesões de luxação durante a infância, sendo a maioria delas curadas espontaneamente se houver um rigoroso cuidado quanto à higiene bucal, porém em traumas mais agudos, pode haver a necessidade de intervenção clínica e/ou cirúrgica $^{13}$. É importante saber que a intensidade do trauma pode ocasionar distúrbios no germe dentário em desenvolvimento, assim como o impacto do dente lesionado, sendo que quanto menos formado estiver o germe do permanente, maior é a chance do mesmo ser afetado devido ao trauma do dente decíduo. A perda precoce de elementos dentários, especialmente os incisivos superiores, é uma grande preocupação não apenas pela situação estética, mas também pelo comprometimento funcional dos dentes ${ }^{14}$.

O traumatismo dental pode ocasionar diversas consequências para os dentes decíduos, tanto para a polpa quanto ao peridonto. Dentre as sequelas que podem ocorrer - mobilidade do elemento dental, alteração de posição na arcada dentária, sintomatologia dolorosa, sensibilidade, hiperemia pulpar, retenção prolongada - a 
necrose pulpar é uma das que mais se destaca, devido à sua complexidade para diagnóstico, que pode ser realizado clinicamente quando o dente apresentar um quadro agudo ou já ter sofrido perda de estrutura dentária; ou radiograficamente, em caso de já ter sofrido perda de estrutura óssea. As reabsorções dentárias podem ou não estar relacionadas à outras alterações, como a necrose pulpar, sendo classificadas em internas ou externas. Para situações de reabsorção leve, só 0 acompanhamento radiográfico é indicado, diferentemente de casos mais severos, onde o tratamento ideal é a exodontia ${ }^{15}$. E assim como o ocorrido neste caso clínico, a descoloração dos dentes decíduos também é uma comum complicação após as lesões de subluxação, podendo apresentar colorações acinzentadas e/ou azuladas após o trauma, decorrentes da hemorragia pulpar ${ }^{11}$. Dentes que persistem com descoloração escurecida meses ou anos após o incidente podem permanecer clínica e radiograficamente assintomáticos, sendo possível que no futuro evolua para a necrose da polpa. Da mesma forma, os dentes também podem apresentar descoloração transitória avermelhada e/ou amarelada, indicando, neste último caso, obliteração (calcificação) do canal pulpar, caracterizado pela deposição de tecido mineralizado no interior do canal radicular ${ }^{9}$.

Em casos de subluxação, é essencial diagnosticar a direção do deslocamento do dente para descartar a possibilidade de lesão ao sucessor, evitando que ocorram sequelas na futura dentição. $O$ cuidado com a prescrição de antibioticoterapia também é necessário para impedir a propagação da inflamação para o germe do sucessor permanente. No presente caso, a exodontia foi 0 melhor plano de tratamento instituído, visto os achados clínicos (alteração de cor relacionada à lesão, presença de fístulas e mobilidade dentária) e radiográficos (reabsorção radicular extensa dos decíduos, rompimento de cripta óssea do germe dos permanentes e lesão peiapical - diagnóstico de necrose pulpar), sendo evidente a irregularidade da superfície radicular reabsorvida após a extração ${ }^{16}$. Para traumas de subluxação, também recomenda-se o cuidado com a higiene bucal, devendo ser realizada a limpeza dos dentes com escova dental macia e aplicação tópica de clorexidina $0,12 \%$ com o auxílio de cotonete duas vezes ao dia por uma semana. Além do cuidado com a higiene, faz-se necessária uma mudança dietética, com alimentos pastosos e líquidos nos 2 primeiros dias após o trauma, além da remoção de hábitos de sucção não-nutritivos (mamadeira e chupeta). Contenções também podem ser utilizadas em torno de 2 semanas para manter os dentes em posição de repouso, auxiliando no reparo do feixe vasculonervoso e das fibras periodontais, além de fornecer maior conforto ao paciente. $\mathrm{O}$ odontopediatra pode lançar mão da contenção flexível (fio de náilon) quando há lesões nos tecidos de sustentação, contenção semirrígida quando há fraturas de tecido ósseo ou contenção rígida quando ocorrem fraturas radiculares $^{4}$. Ainda pode-se optar pelo tratamento endodôntico, mas só em casos onde há presença de infecção associada. Se após o período de observação, entre 6 a 8 semanas após o trauma, a descoloração do elemento dental estiver presente $e$ associada ao desenvolvimento de fistulas, faz-se necessária uma conduta mais rigorosa, assim como o tratamento indicado para 0 presente caso: exodontia ${ }^{9}$.

Com maior conhecimento de eventuais sequelas causadas por traumatismos, os odontopediatras podem compreender mais aprofundadamente como tratar dentes em situações de lesões traumáticas e como lidar com suas possíveis complicações, sendo apenas através de avaliações clínicas e radiográficas que essas conclusões podem ser verificadas. Os resultados obtidos através de exames clínicos e complementares são capazes de fornecer diretrizes para auxiliarem os profissionais, delineando uma abordagem eficaz para o atendimento imediato ou de urgência na clínica odontológica, aumentando as chances de um bom prognóstico.

CONCLUSÃO

Podemos compreender com este relato de caso que é extremamente necessário que o cirurgião-dentista tenha conhecimento acerca dos possíveis traumatismos que possam ocorrer na infância e seus respectivos protocolos de atendimento frente às lesões decorrentes do trauma. Além de ser imprescindível que o mesmo esteja comprometido em propagar medidas preventivas para evitar ou diminuir os casos de traumatismos recorrentes.

\section{REFERÊNCIAS}

1. Goettems ML, Torriani DD, Hallal DC, Correa MB, Demarco FF. Dental Trauma: Prevalence and Risk Factors in Schoolchildren. Community Dent Oral Epidemiol. 2014;42:581-590.

2. Barros JNP, Araújo TAA, Soares TRC, Lenzi MM, Risso PA, Fidalgo TKS, et al. Profiles of Trauma in Primary and Permanent Teeth of Children and Adolescents. J Clin Pediatr Dent. 2019;43:5-10.

3. Sanabe ME, Cavalcante LB, Coldebella CR, Lima FCBA. Urgências em traumatismos 
dentários: classificação, características e procedimentos. Ver Paul Pediatr. 2009;27: 447-51.

4. Emerenciano NG, Santin GC, Calvo ML, Terra GMO, Martioli G, Provenzano MGA. Traumatismo na dentição decídua: um relato de caso clínico. Arch Health Invest. 2015;4:25-350.

5. James V, Vandersluis YR, Zhang EWJ, Scolnik D. Dental Injuries in Younger Emergency Department Patients. CJEM. 2018;20:425-31.

6. O'Neill, G. Dental Trauma: Trauma Protocol for Schools. Br Dent J. 2018;225:376.

7. Alhaddad B, Rózsa NK, Tarján I. Dental Trauma in Children in Budapest. A Retrospective Study. Eur J Paediatr Dent. 2019;20:111-15.

8. Cohenca N. Traumatic Dental Injuries: Adherence to Treatment Guidelines Critical to Positive Patient Outcomes. Rev Assoc Paul Cir Dent. 2016;70:386-97.

9. Malmgren B, Andreasen JO, Flores MT, Robertson A, DiAngelis AJ, Andersson L, et al. Guidelines for the Management of Traumatic Dental Injuries: 3. Injuries in the Primary Dentition. Pediatr Dent. 2013;39:420-28.

10. Tewari N, Bansal K, Mathur VP. Dental Trauma in Children: A Quick Overview on Management. Indian J Pediatr. 2019;86:1043-47.

11. Silveira ALNMS, Magno MB, Soares TRC. The Relationship Between Special Needs and Dental Trauma. A Systematic Review and Meta-Analysis. Dent Traumatol. 2020;36: 218-36.

12. Khan L. Dental Care and Trauma Management in Children and Adolescents. Pediatr Ann. 2019;48:3-8.

13. Lenzi MM, Fidalgo TKS, Luiz RR, Maia LC. Trauma in Primary Teeth and Its Effect on the Development of Permanent Successors: A Controlled Study. Acta Odontol Scand. 2019;77:76-81.

14. Magno MB, Neves AB, Ferreira DM, Pithon MM, Maia LC. The Relationship of Previous Dental Trauma With New Cases of Dental Trauma. A Systematic Review and MetaAnalysis. Dent Traumatol. 2019;35:3-14.

15. Ozdas DO, Zorlu S, Karataban PK. Traumatic Dental Injury Incidence of Unseen Children. Dent Traumatol. 2019;35:15-9.

16. Wallace A, Rogers HJ, Zaitoun H, Rodd HD, Gilchrist F, Marshman Z. Traumatic Dental Injury Research: On Children or With Children? Dent Traumatol. 2017;33:153-59.

\section{CONFLITO DE INTERESSES}

Os autores declaram não haver conflitos de interesse

\section{AUTOR PARA CORRESPONDÊNCIA}

Marcele Danelon
Departamento de Odontologia,
Universidade de Ribeirão Preto - UNAERP
Av Costábile Romano 2.201
14096-900 Ribeirão Preto - SP, Brasil
Tel. +55 1636036717
E-mail: marcelledanelon@hotmail.com

Submetido em 20/06/2020 Aceito em 23/10/2020 